\title{
Amplitude Noise Suppression and Orthogonal Multiplexing Using Injection-Locked Single-Mode VCSEL
}

\section{Lyubopytov, Vladimir S.}

OSA Publishing

2017

Lyubopytov , V S, von Lerber , T, Lassas , M , Malekizandi , M , Chipouline , A \& Kueppers , F 2017 , Amplitude Noise Suppression and Orthogonal Multiplexing Using Injection-Locked Single-Mode VCSEL . in OPTICAL FIBER COMMUNICATIONS CONFERENCE 2017 . vol. 2017 , OSA Publishing , The Optical Fiber Communication Conference and Exhibition (OFC) , Los Angeles , United States , 19/03/2017 . https://doi.org/10.1364/OFC.2017.Tu3C.3

http://hdl.handle.net/10138/311063

https://doi.org/10.1364/OFC.2017.Tu3C.3

unspecified

acceptedVersion

Downloaded from Helda, University of Helsinki institutional repository.

This is an electronic reprint of the original article.

This reprint may differ from the original in pagination and typographic detail.

Please cite the original version. 


\title{
Amplitude Noise Suppression and Orthogonal Multiplexing Using Injection-Locked Single-Mode VCSEL
}

\author{
Vladimir S. Lyubopytov ${ }^{1,2,3}$, Tuomo von Lerber ${ }^{2,4}$, Matti Lassas ${ }^{4}$, Mohammadreza Malekizandi², \\ Arkadi Chipouline ${ }^{2}$, Franko Küppers ${ }^{2}$ \\ 1 Dpt. Photonics Engineering, Technical University of Denmark (DTU), Akademivej Building 343, 2800 Kgs. Lyngby, Denmark \\ 2 Institute for Microwave Engineering and Photonics, Technische Universität Darmstadt, 64283 Darmstadt, Germany \\ 3 Telecommunication Systems Dept., Ufa State Aviation Technical University, 12 K. Marx St., 450000 Ufa, Russia \\ 4 Department of Mathematics and Statistics, University of Helsinki, P.O. Box 68 (Gustaf Hällströmin katu 2b)FI-00014 Helsinki, Finland \\ E-mail: lyubopytov.v.ru@ieee.org
}

\begin{abstract}
We experimentally demonstrate BER reduction and orthogonal modulation using an injection locked single-mode VCSEL. It allows us suppressing an amplitude noise of optical signal and/or double the capacity of an information channel.

OCIS codes: (140.7260) Vertical cavity surface emitting lasers; (060.5060) Phase modulation; (030.1670) Coherent optical effects
\end{abstract}

\section{Introduction}

Phase locking of a laser with an external seeding signal has been investigated in details [1], and stable locking conditions have been determined [2]. In spite of the multiple publications devoted to this problem, the case of phasemodulated external seeding has been rather rarely considered. It has been found, that the laser under certain conditions can follow the phase of the external seeding, while keeping its own output amplitude, almost independent from the input seeding signal [3].

It has been shown earlier, that the injection locked semiconductor lasers enable an effective amplitude noise suppression [4] and makes possible an extra level of signal multiplexing - orthogonal modulation [5], where PSK and ASK NRZ channels propagate at the same wavelength [6].

In this work we use for the first time to our knowledge a VCSEL as a slave laser providing amplitude disturbance suppression and consequently BER improvement. VCSELs are known to have high energy efficiency due to small threshold currents, low fabrication cost, possibility of on-chip integration, and effective coupling with an optical fiber.

An injection locked VCSEL can extract the phase information before receiver effectively suppressing the amplitude modulation, which the phase modulated signal acquires propagating through the dispersive fiber. As a result, the BER decreases significantly

Also amplitude and phase modulations, carrying independent information flows (orthogonal modulation [5, 6]), have been separated using an injection locked VCSEL. This approach can be used as a cost- and energy-efficient extension of the existing systems in order to improve the capacity of short-range optical fiber communications and optical interconnects for data centers. This method is simple, requires only relatively small modification in infrastructure, and hence is supposed to be cost effective.

An extra advantages can be potentially provided in future by wavelength tunable MEMS-VCSELs [7]. The tunability of the MEMS-VCSEL could allow us to control precisely the wavelength detuning between the seeding signal and free run mode of the MEMS-VCSEL.

\section{Operation principle and experimental setup}

To validate the possibility of phase modulation extraction by an injection-locked VCSEL, an experimental setup shown in Fig. 1 has been built. CW light from a tunable external cavity laser (ECL), used as a master laser, is consecutively modulated by amplitude and phase with $1 \mathrm{~Gb} / \mathrm{s}$ rate. An electro-absorption modulator (EAM) and phase modulator (PM) are independently fed by two pulse pattern generators (PPG), which provide pseudorandom binary sequences (PRBS) with lengths of $2^{9}-1$ and $2^{7}-1$ bits respectively. PPGs are synchronized in such a way that the modulated amplitude and phase symbols in optical signal coincide in time. This part emulates propagation of the signal through the dispersive fiber with appearance of amplitude modulation and losses modeled by attenuator. The amplified spontaneous emission (ASE) noise of optical amplifiers is introduced in order to test different OSNR scenarios in a hypothetic transmission system. After EDFA and 3-nm optical bandpass filter, the received signal passes through the variable attenuator, which allows optimizing an injection power. An optical circulator is used to separate the injected and emitted by VCSEL signals. The VCSEL (slave laser) is pumped slightly above its lasing 
threshold. Polarization controller before the VCSEL provides the optimal polarization state of the injected optical signal. The output signal of VCSEL after the circulator is detected by a differential binary phase-shift keying (DBPSK) receiver. Analysis of the detected bit sequences is performed by the bit error rate tester (BERT). For capturing eye diagrams and measuring extinction ratio (ER) before and after VCSEL, digitizing oscilloscope is used.

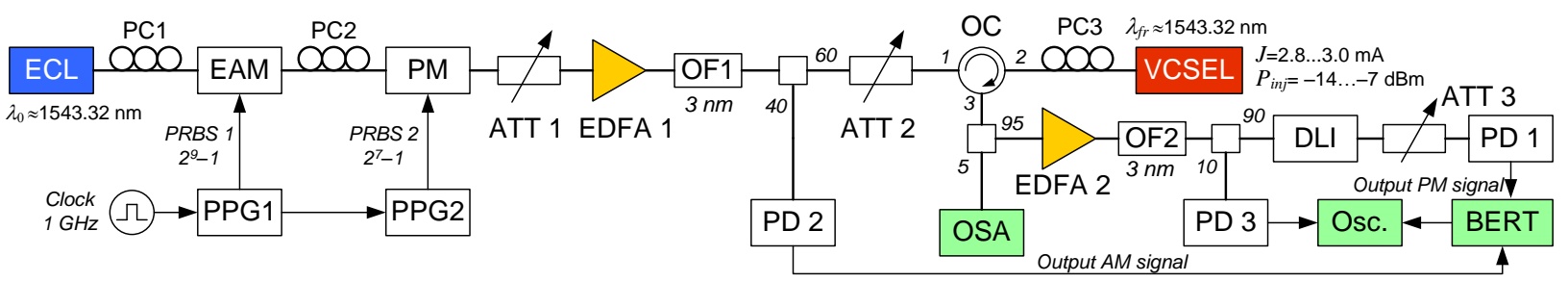

Fig. 1. Scheme of the experimental setup (ATT - variable attenuator; OF - 3-nm band pass optical optical filter; PD - p-i-n photodiode; DLI delay line interferometer; OSA - optical spectrum analyzer; Osc. - digitizing oscilloscope).

The desirable regime of injection locking can be obtained when the detuning between master and slave lasers approaches zero (from the shorter wavelengths). The developed recently MEMS-VCSELs [7] could therefore play a crucial role in the proposed technique, allowing accurate wavelength matching. In our experimental setup an absolute value of detuning was first minimized coarsely by setting the wavelength of ECL as close as possible to the free-running wavelength of VCSEL, and then optimized more precisely by changing the temperature and driving current of the VCSEL. Depending on both VCSEL current and resulting detuning, an optimal injection power was empirically adjusted in order to minimize BER in the demodulated signal after DBPSK receiver. It follows from the experimental observations that for optimal regime of phase translation and amplitude modulation suppression an external power injection ratio has to be $>14 \mathrm{~dB}$.

\section{Experiment results and discussion}

The obtained results shown in Fig. 2(a) demonstrate the suppression of the amplitude modulation (AM) in an optical signal rectified by the VCSEL. At the value of $E R=7.8 \mathrm{~dB}$, where eye diagram of the detected DBPSK-signal tends to be closed, employment of the proposed approach provides gain of $4.75 \mathrm{~dB}$ in diminishing ER, which allows keeping the eye open. When the ER of AM in the input signal approaches $10 \mathrm{~dB}$, the use of VCSEL provides ER suppression of $6.37 \mathrm{~dB}$. Fig. 2(b) shows BER vs. AM ER dependences. As follows from these curves, utilization of the injection-locked VCSEL provides significant improvement of the transmission reliability for DBPSK signal component, BER improvement of 8 orders can be achieved. Consequently, after VCSEL-based rectification the impairment caused by amplitude modulation due to acquired dispersion is no more limiting factor of the system.
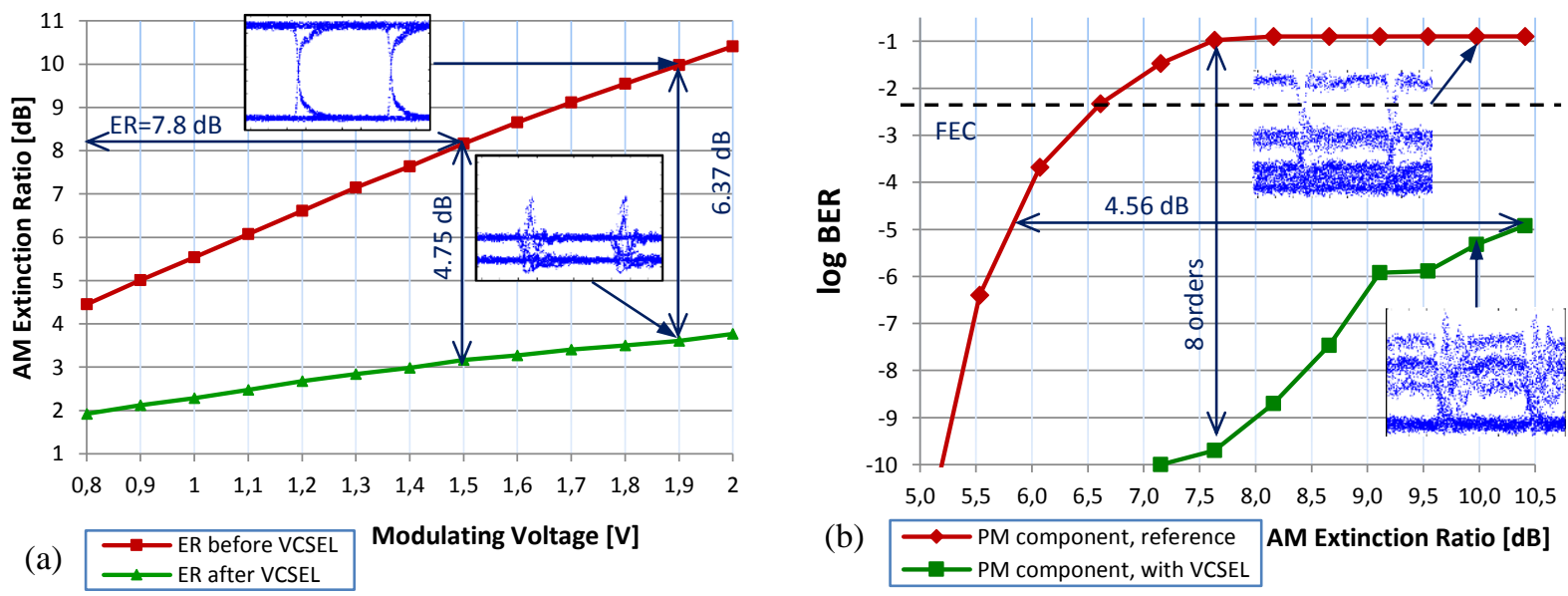

Fig. 2. (a) Extinction ratio of amplitude modulation measured at the input (red) and the output (green) of injection-locked VCSEL; (b) BER vs. extinction ratios of amplitude modulation for the DBPSK channel before (red) and after (green) phase rectification using VCSEL.

Efficiency of suppression of the amplitude modulation by injection-locked laser depends on its time scale. The maximum treatable symbol rate of AM is limited by the time required for damping relaxation oscillations and establishing a steady-state regime. For the VCSEL used in our setup, relaxation oscillations take about $0.4 \mathrm{~ns}$, so that the optical signal with a symbol rate not higher than $2.5 \mathrm{Gbit} / \mathrm{s}$ could be processed. However, state-of-art VCSELs with enlarged bandwidth [8] could be used for high speed transmission. 
In the scenario of simultaneous orthogonal amplitude and phase modulation, BERT was connected to the $40 \%$ output of the fiber splitter after EDFA 1 to analyze the bit sequence encoded by the optical signal amplitude, and to the output of DBPSK receiver to analyze the phase-modulated component. Fig. 3 shows BER vs. AM ER dependences for the amplitude- and phase-modulated signals (orthogonal modulation) in the presence of ASE for losses in the transmission line of $-12 \mathrm{~dB}$. The utilization of the injection-locked VCSEL provides significant improvement of the transmission reliability for DBPSK signal component; moreover, the higher initial ER of AM, the more BER improvement can be achieved.

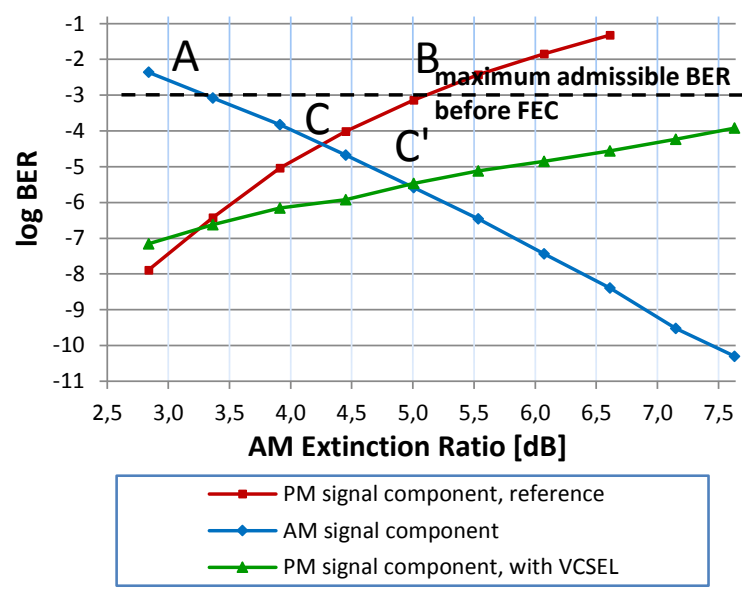

Fig. 3: BER vs. ER of amplitude modulation for the amplitude-modulated channel (blue), and the DBPSK channel before (red) and after (green) phase rectification using VCSEL. Points A and B are the crossing with the maximum admissible BER before FEC. Crossing points C and C' are the points of equal BERs for amplitude- and phase-modulated channels - improvement of more than an order is recognized.

The use of the injection-locked VCSEL as an optical phase rectifier gains BER with a simple differential phase demodulation scheme even for quite high ERs of amplitude modulation, superimposed over the phase one.

\section{Conclusions}

We have found that the special operation mode of an externally seeded VCSEL can be used for effective suppression of the residual amplitude modulation in phase-modulated optical signal. Based on this effect, we have successfully demonstrated BER improvement of the phase modulated signal by several orders of magnitude. It is believed that the proposed technique can find immediate applications for the fiber optic coherent transmission lines. We have shown experimentally that the special operation mode of an injection locked VCSEL can be used for effective separation of the phase and amplitude modulated signals simultaneously carried by the same wavelength orthogonal modulation. We have demonstrated (de)modulation scheme, which provides at least double information capacity increase using the standard communication system architecture by the cost of one extra element (VCSEL) per wavelength channel.

V. S. L. acknowledges the support of the German Academic Exchange Service (DAAD) and the Ministry of Education and Science of Russian Federation within the "Michail Lomonosov" programme.

\section{References}

[1] H. Haken, Laser Light Dynamics, Vol. 2 (North-Holland Physics Publishing, 1986).

[2] E. K. Lau, L. J. Wong, and M. C. Wu, "Enhanced Modulation Characteristics of Optical Injection-Locked Lasers: A Tutorial," IEEE J. Sel. Topics Quantum Electron. 15(3), 618-633 (2009).

[3] A. Pikovsky, M. Rosenblum and J. Kurth, Synchronization. A Universal Concept in Nonlinear Science (Cambridge University Press, 2001), p. 46.

[4] A. Fragkos, A. Bogris, D. Syvridis, and R. Phelan, "Colorless Regenerative Amplification of Constant Envelope Phase-Modulated Optical Signals Based on Injection-Locked Fabry-Pérot Lasers,” IEEE Photon. Technol. Lett. 24(1), 28-30 (2012).

[5] Y. Zhang, N. Deng, C.-K. Chan, and L.-K. Chen, "A Multicast WDM-PON Architecture Using DPSK/NRZ Orthogonal Modulation", IEEE Photonics Technology Letters, 20, 1479, 2008.

[6] A. Fragkos, A. Bogris, and D. Syvridis, "Spectrally efficient and High Extinction Ratio DPSK/ASK Orthogonal Modulation Schemes Based on Injection Locking Limiting Amplifiers", in ECOC Technical Digest, (OSA, 2012), paper P3.04.

[7] S. Paul, C. Gierl, J. Cesar, Q. T. Le, M. Malekizandi, B. Kögel, C. Neumeyr, M. Ortsiefer, F. Küppers, "10 Gbit/s Direct Modulation of Widely Tunable $1550 \mathrm{~nm}$ MEMS VCSEL”, IEEE J. Sel. Topics Quantum Electron. 21(6), (2015).

[8] D. M. Kuchta A. V. Rylyakov, F. E. Doany, C. L. Schow, J. E. Proesel, C. W. Baks, P. Westbergh, J. S. Gustavsson, and A. Larsson, “A 71-Gb/s NRZ Modulated 850-nm VCSEL-Based Optical Link,” IEEE Photon. Technol. Lett. 27(6), 577-580 (2015). 